\title{
Target Tracking Control of Mobile Robot Based on Ultrasonic Sensor
}

\author{
Zhenwen $\mathrm{Tan}^{1, \mathrm{a}}$, Shusheng $\mathrm{Bi}^{2, \mathrm{~b}}$, Hao Wang ${ }^{2, \mathrm{c}}$ and Zhibiao Wang ${ }^{2}$ \\ ${ }^{1}$ School of Mechanical Engineering \& Automation, Beihang University, Beijing 100191, China; \\ ${ }^{2}$ School of Mechanical Engineering \& Automation, Beihang University, Beijing 100191, China. \\ atanzhenwen_buaa@163.com, bssbi@buaa.edu.cn, cwmy670926@163.com
}

Keywords: Target Tracking, Ultrasonic Sensor, Angle Gradient, Smoothly

\begin{abstract}
Design and realize an intelligent mobile robot system applied to track the target using ultrasonic sensor. Wheeled mobile robot senses the orientation between robot and target through the radio device and ultrasonic sensor, and then combines with target-tracking algorithm track following algorithm and angle gradient algorithm, eventually achieves the objective that following the target. In order to expand the reception of ultrasound and enhance the positioning accuracy, the ultrasonic sensors are installed in a special way. The experiment result indicates that the mobile robot can move smoothly and follow the target in real time.
\end{abstract}

\section{Introduction}

At present, wheeled mobile robots are being given much attention in recent literature. Mobile robots are increasingly used in manufacturing industry and in service industry, for domestic needs and also for entertainment, but there is still no many robot product possessing the function of target-tracking on the domestic market [1, 2, 3]. Nowadays, most of robots follow the target through the pathway given before. Furthermore these products are mainly applied to assembly line work, not suitable for families, supermarkets, outdoors and other common occasions [4]. In order to realize the function, wheeled mobile robot following the specified target more smoothly in real time, this article designs a robot system using ultrasonic sensor and proposes a target-tracking algorithm.

This paper is organized as follows. Section 2 presents the structure and principle of system. The installation of ultrasonic and position principle is shown in Section 3. The target-tracking controller is designed in Section 4. In Section 5, some results and discussion from computer practical experiment are given to verify the tracking controller. The conclusion remark is given in section 6 .

\section{Structure and Principle of System}

This article adopts a typical four-wheeled mobile robot as mechanical platform. Two differentially driven rear wheels and two passive self-adjusted supporting wheel (castor front wheel) constitute the mechanical structure.

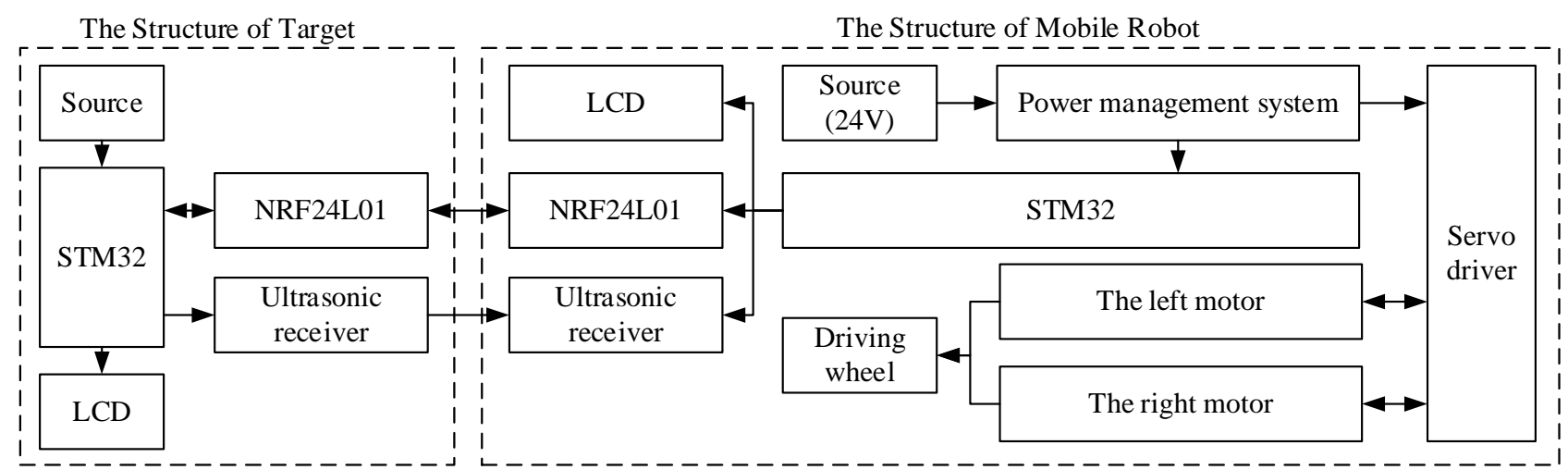

Fig. 1 Structure of the system

Main structure of this system can be given by Fig 1 . To achieve the aim that mobile robot track the target, we design a specific ultrasonic location system where the ultrasonic transmitter and receivers 
are separated. Ultrasonic transmitter is carried by target, and a number of ultrasonic receivers are placed on the mobile robot. And two nRF24L01 wireless modules are individually fixed on the target and robot. Firstly, mobile robot transmit radio signal, then the target emit ultrasonic signal after receiving the radio signal, and finally the robot start timing so that we can measure the distance between ultrasonic transmitter and receivers.

The wheeled mobile robot employ STM32 microcontroller as microprocessor, and emit a radio signal to target every $30 \mathrm{~ms}$ to detect the distance in real time. Based on these distances, we can calculate the target's location in robot coordinate. And that, utilize target-tracking algorithm to control speed of driven wheels. Ultimately, the state of robot motion and the position of target is shown on LCD. Fig. 2 shows the feature of the mobile robot of which size is $50 \mathrm{~cm} \times 50 \mathrm{~cm} \times 50 \mathrm{~cm}$. It has the same structure as in Fig. 5, with two driving wheels and two passive centered passive self-adjusted supporting wheels. In addition, the ultrasonic transmitter that carried by the target is shown in Fig. 3.

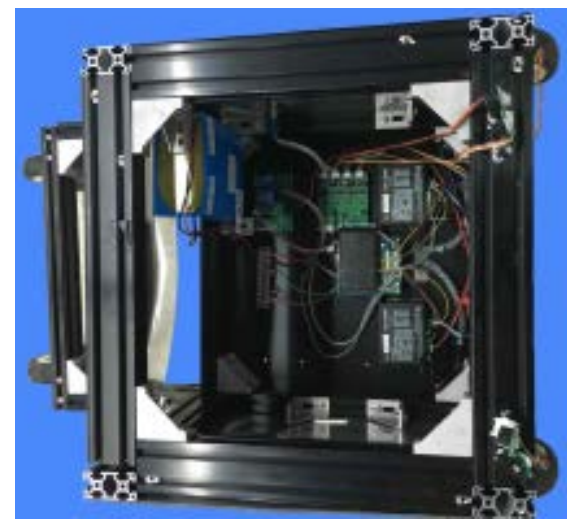

Fig. 2 The wheeled mobile robot

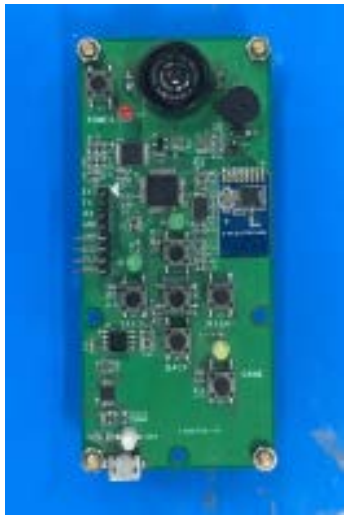

Fig. 3 The ultrasonic transmitter

\section{Positioning principle}

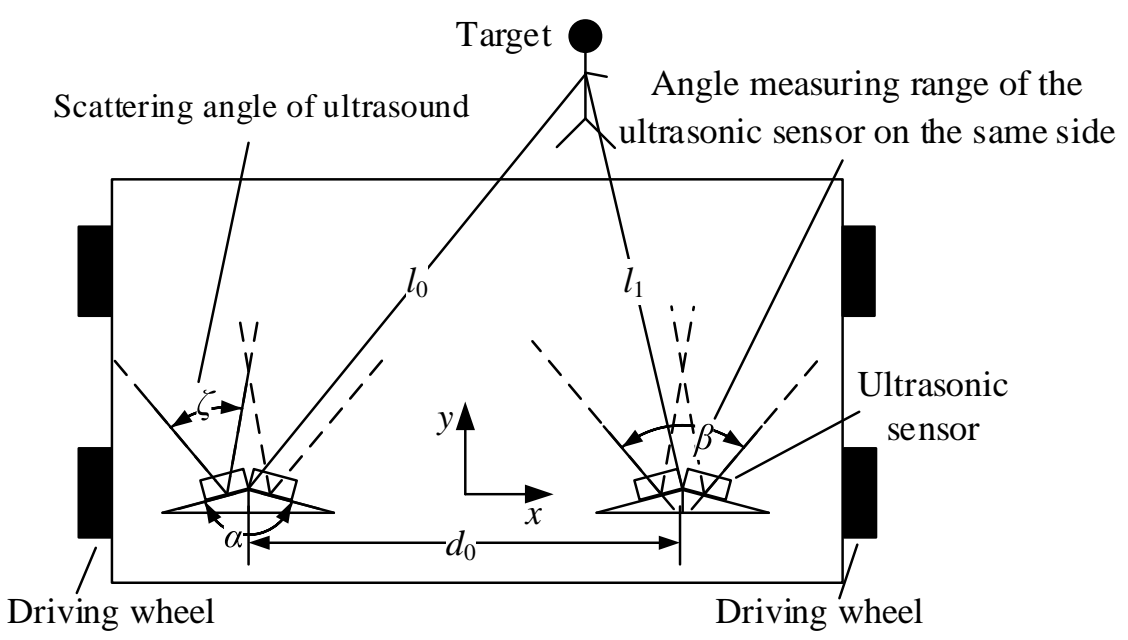

Fig. 4 The installation method of ultrasonic sensors

The measurement range of ultrasonic sensor is limited because of the presence of ultrasound scattering angle $[5,6]$. By do some experiments, we can measure that the scattering angle $(\zeta)$ of ultrasonic sensor is $60^{\circ}$. So we propose an installation method to expand the measuring range. As is shown in Fig 4, two sensors on each side are attached to the axis of driving wheels symmetrically, in addition, the sensor at one side are installed at an angle of $\alpha$ degrees $\left(130^{\circ}\right)$. The distance between left side and right side is $d_{0}$. This method of installation expand the measurement range of sensors on the same side from $\zeta$ to $\beta$, consequently, the situation where robot cannot detect the position of target rarely appear. 
According to the above method, we can obtain four distances between ultrasonic transmitter and receivers. As is shown in Fig. 4, if both of sensors on same side can receive the signal send by ultrasonic transmitter, $l_{0}$ equals to the mean of the two distances. Otherwise, $l_{0}$ is equal to the distance detected by sensor that can receive ultrasonic signal. $l_{1}$ is measured in the same way.

Through the geometric relationships shown in Fig 4, we can obtain the target's position in the robot coordinate system, where they are written as [7]:

$$
\left\{\begin{array}{l}
x=\frac{l_{0}^{2}-l_{1}^{2}}{2 \cdot d_{0} \cdot l_{0}} \\
y=l_{0} \cdot \sqrt{1-x^{2}}
\end{array}\right.
$$

It was converted into a polar coordinate system, we can conclude: $\theta=\arctan y / x$ and $\rho=\sqrt{ }\left(x^{2}+y^{2}\right)$.

\section{Target Tracking}

\subsection{Principle of Tracking}

After positioning the target, we will planned a path to reach the target smoothly in real time. In this paper, adopt the way of TWT (Turn While Travelling) to make robot follow the target [8]. As is shown in Fig 5, the mobile robot's trajectory that robot arriving at the target is arc-shaped, and velocity is proportional to the distance between robot and target. Establish the robot Cartesian coordinate, where $r$ is instantaneous curvature radius of the robot trajectory, $d$ is distance between two driven wheels. $\rho$ equals the distance between target and mobile robot, $\theta$ is the direction angle, $v_{\text {left }}$ and $v_{\text {right }}$ denote the linear velocities of two driving wheels. $v_{o}$ equals average value of $v_{\text {left }}$ and $v_{\text {right }}[9,10$, $11]$.

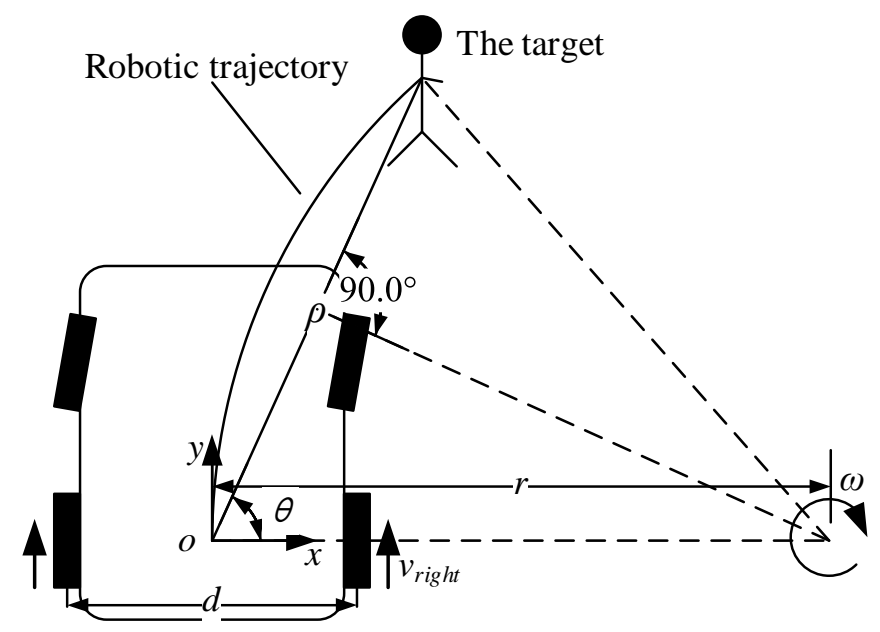

Fig. 5 The principle of trajectory tracking

According to principles of geometry, kinematical equation of differential steering mobile robot can be presented as:

$$
\left\{\begin{array}{l}
v_{\text {left }}=\omega(r+d / 2) \\
v_{\text {right }}=\omega(r-d / 2) \\
v_{o}=\left(v_{\text {left }}+v_{\text {right }}\right) / 2
\end{array}\right.
$$

And through the equation (2), the value of $v$ and $\omega$ can be obtained as follows:

$$
\left\{\begin{array}{l}
\omega=\frac{v_{\text {left }}-v_{\text {right }}}{d} \\
r=\frac{d}{2} \cdot \frac{v_{\text {left }}+v_{\text {rigt }}}{v_{\text {left }}-v_{\text {right }}}
\end{array}\right.
$$

Obviously, the relationship between $r$ and $\rho$ is described as: $r=\rho /(2 \cos \theta)$. And we assume that the relation between $v_{o}$ and $\rho$ is in equation (4). 
$v_{o}=f(\rho)=0.5 \rho$

Depending on equation (3) and (4), the speed of motor is considered as controlled quantity, so the input parameter of mobile robot can be written as:

$$
\left\{\begin{array}{l}
v_{\text {left }}=(1+d / \rho \cdot \cos \theta) \cdot 0.5 \rho \\
v_{\text {right }}=(1-d / \rho \cdot \cos \theta) \cdot 0.5 \rho
\end{array}\right.
$$

\subsection{Angle Gradient}

The position of target may change sharply in robot coordinate as a result of low speed of ultrasound and uncertainty of target velocity. This can lead tremble of robot, especially the change of $\theta$. So we adopt way of angle gradient to make robot follow the target more smoothly.

Since the low speed of ultrasound and uncertainty of target velocity, we adopt the way of angle gradient to make robot follow the target more smoothly. As is shown in Fig 6, $\theta_{\text {pre }}$ is the last measured value. If $\mid \theta$ - $\theta_{\text {pre }} \mid>10$, then $\theta=\theta+3$ or $\theta=\theta$ - 3, in other words, $\theta$ changes slowly.

The experiment shows this method is real-time and efficient and make robot move more smoothly.

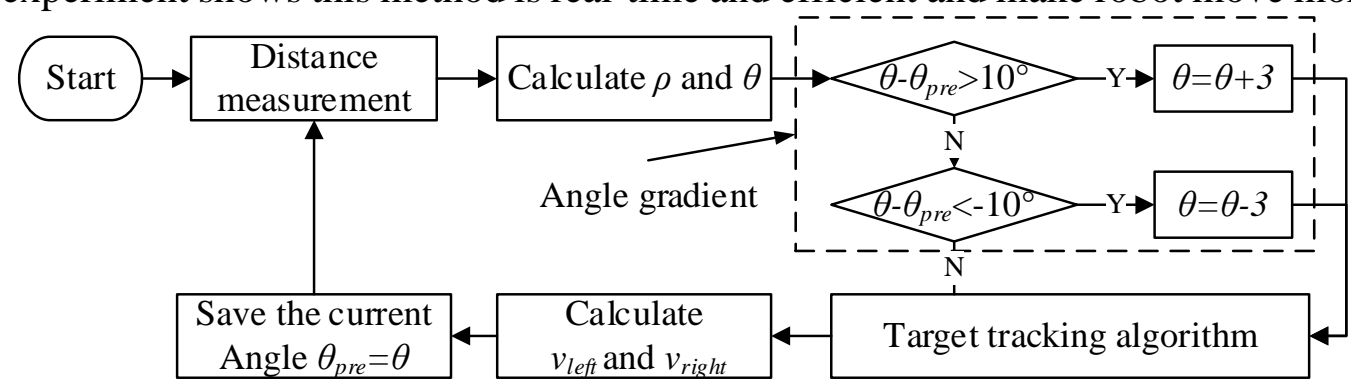

Fig. 6 The system flow chart

\section{Result of Experiments}

The most common trajectory is straight line and arc while wheeled mobile robot is in motion. So the proposed algorithm is verified with experiments that follow the two trajectories. The proposed algorithm is verified with experiments that robot follows the target moving in a straight line or in circular motion.

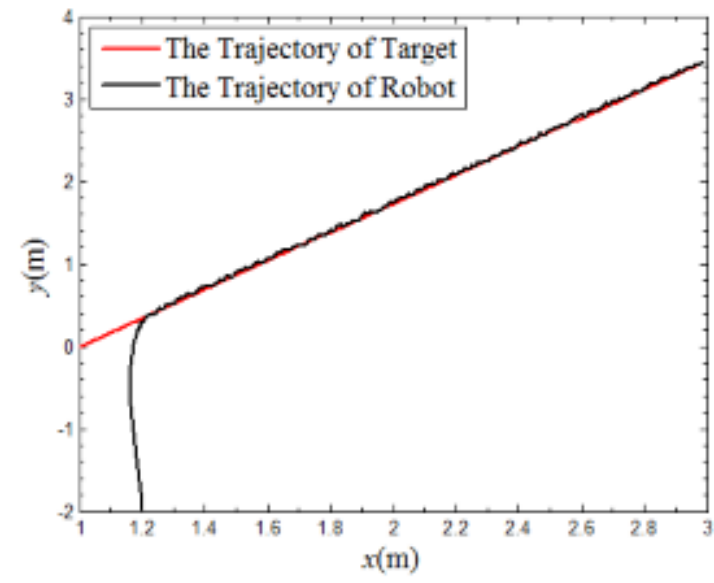

Fig. 7 Trajectory tracking of straight line

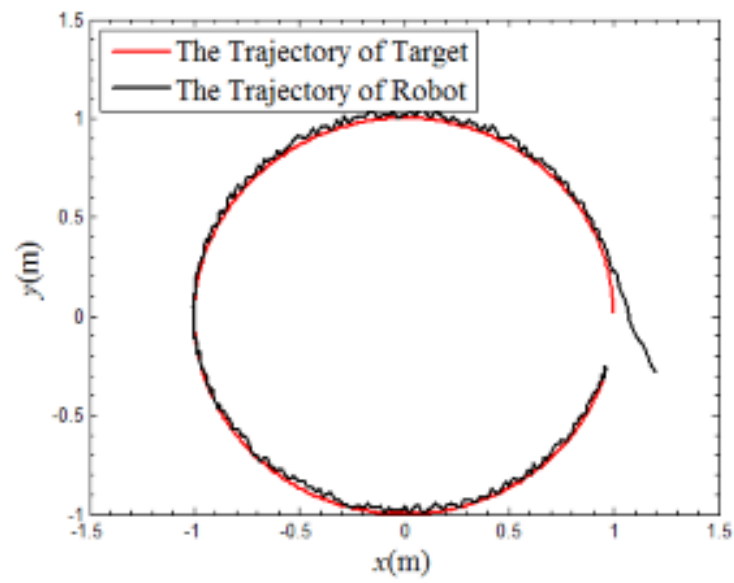

Fig. 8 Trajectory tracking of circle

Fig. 7 shows the result of experiment tracking the target with walking straight at speed of $0.5 \mathrm{~m} / \mathrm{s}$. The trajectory of target is $y=\sqrt{3} x-\sqrt{3}$.The initial positions of target and robot are $(1 \mathrm{~m}, 0 \mathrm{~m})$ and $(1.2 \mathrm{~m},-2 \mathrm{~m})$. And y-axis positive direction of robot towards the target. Fig. 8 indicates the result of experiment tracking the target in circular motion. The target's trajectory is defined by rotating at a constant linear speed of $0.8 \mathrm{~m} / \mathrm{s}$ along a circular path of radius $1 \mathrm{~m}$ with its center located at $(0 \mathrm{~m}, 0 \mathrm{~m})$. Its starting point is at $(1 \mathrm{~m}, 0 \mathrm{~m})$. The initial position of robot is $(1.2 \mathrm{~m},-0.3 \mathrm{~m})$. The experiments show that mobile robot can follow the target smoothly in real time. 


\section{Summary}

In this paper, a new way of positioning using ultrasonic sensor and a novel target tracking algorithm are proposed to solve the tracking problem of mobile robot. And the mobile robot can track the target smoothly in real time. What's more, the corresponding experiments result demonstrates the effectiveness of the algorithm.

It was found that the mobile robot occasionally deviate from the direction of the target in the travel process. One of the reasons is the presence of ultrasound reflection, the other is the measurement range of ultrasonic sensor is restricted in spite of using the new installation method. These lead the target is located inaccurately or not located completely. In the future, in order to positioning the target in all directions, we can introduce radiolocation to take the place of ultrasonic sensor.

\section{References}

[1]. X.Z. Wang. Design of Tracking Robot Based on Infrared Sensor and Ultrasonic Sensor (MS, Harbin Institute of Technology, China 2009). p. 9.

[2]. D.T. Tan. Research and design of human tracking robot (MS, Northwest Normal University, China 2013). p. 32.

[3]. J. Jin. Research of tracking and obstacle avoidance control for autonomous mobile robot (MS, Hunan University, China 2014). p. 2.

[4]. Z.Y. Lu. Design of autonomous navigation mobile robot (MS, Nanchang University, China 2013). p. 6.

[5]. Y.R. Song, D.Y Li, W.C Cai. Mobile Robots and the Autonomous Technologies. China Machine Press, 2012, p. 152-158.

[6]. L. Liu, X.F Sun, Y. Zhang. Remote controlling and intelligent following car design based on STM32. Electronic Measurement Technology. Vol. 38(2015) No.6, p. 31-33.

[7]. L. Cai, T.T. Zhou, Y.P. Guo. Intelligent Following Carriage Based on Ultrasonic Positioning. Electronic Measurement Technology. Vol. 36(2013) No.11, p. 76-79.

[8]. Gerald Cook, et al. Mobile Robots: Navigation, Control and Remote Sensing. National Defend Industry Press, 2015, p. 17-18.

[9]. Hamerlain F, et al. Trajectory tracking control of a car-like mobile robot in presence of sliding. UKACC International Conference on Control. Cardiff University, UK, 2012, p.502-507.

[10]. Ni N, Jia Y, Du J, et al. Non-fragile control for trajectory tracking of nonholonomic mobile robots. American Control Conference (ACC). Chicago, USA, 2010, p. 214 - 218.

[11]. L. Liu, P. Xiang, Y.J. Wang. Trajectory tracking of a nonholonomic wheeled mobile robot. Journal of Tsinghua University (Science and Technology). Vol. 47(2007) No. S2, p. 1884-1889. 